\title{
1 Ensemble cryo-electron microscopy reveals conformational 2 states of the nsp13 helicase in the SARS-CoV-2 helicase 3 replication-transcription complex
}

5 James Chen ${ }^{1,6}$, Qi Wang ${ }^{2,6}$, Brandon Malone ${ }^{1,6}$, Eliza Llewellyn ${ }^{1}$, 6 Yakov Pechersky ${ }^{2}$, Kashyap Maruthi ${ }^{3}$, Ed T. Eng ${ }^{3}$, Jason K. Perry ${ }^{4}$, 7 Elizabeth A. Campbell ${ }^{1}$, David E. Shaw ${ }^{2,5,7}$, Seth A. Darst ${ }^{1,7,8}$

${ }^{1}$ Laboratory of Molecular Biophysics, The Rockefeller University, New York, NY 10065 10 USA.

11 2D. E. Shaw Research, New York, NY 10036 USA.

$12{ }^{3}$ The National Resource for Automated Molecular Microscopy, Simons Electron

13 Microscopy Center, New York Structural Biology Center, New York, NY 10027 USA.

$14{ }^{4}$ Gilead Sciences, Inc., Foster City, CA 94404 USA.

15 5epartment of Biochemistry and Molecular Biophysics, Columbia University, New York, 16 NY 10032 USA.

19 The authors declare no conflict of interest

$21{ }^{6}$ These authors contributed equally to this work.

$22 \quad{ }^{7}$ Correspondence to: David.Shaw@DEShawResearch.com; darst@rockefeller.edu

23 8Lead contact: darst@rockefeller.edu 
The SARS-CoV-2 nonstructural proteins coordinate genome replication and gene expression. Structural analyses revealed the basis for coupling of the essential nsp13 helicase with the RNA dependent RNA polymerase (RdRp) where the holoRdRp and RNA substrate (the replication-transcription complex, or RTC) associated with two copies of nsp13 (nsp132-RTC). One copy of nsp13 interacts with the template RNA in an opposing polarity to the RdRp and is envisaged to drive the RdRp backwards on the RNA template (backtracking), prompting questions as to how the RdRp can efficiently synthesize RNA in the presence of nsp13. Here, we use cryo-electron microscopy and molecular dynamics simulations to analyze the nsp13 ${ }_{2}-\mathrm{RTC}$, revealing four distinct conformational states of the helicases. The results suggest a mechanism for the nsp13 2 -RTC to turn backtracking on and off, using an allosteric mechanism to switch between RNA synthesis or backtracking in response to stimuli at the RdRp active site.

COVID-19, caused by the coronavirus SARS-CoV-2 ${ }^{1,2}$, continues to devastate the world. The viral RNA-dependent RNA polymerase (RdRp, encoded by non-structural protein 12, or nsp12) functions as a holo-RdRp (comprising nsp7/nsp8 $/$ /nsp12) in a replication-transcription complex (holo-RdRp + RNA, or RTC) to direct RNA synthesis from the viral RNA genome ${ }^{3-5}$. The RdRp is also a target for the clinically approved antiviral remdesivir ${ }^{6-8}$. In addition to the holo-RdRp, the virus encodes several nucleic acid processing enzymes, including a helicase (nsp13), an exonuclease (nsp14), an endonuclease (nsp15), and methyltransferases (nsp14 and nsp16) ${ }^{9}$. Little is known about how these enzymes coordinate to replicate and transcribe the viral genome.

Nsp13, essential for viral replication ${ }^{10-13}$, is a superfamily 1B (SF1B) helicase that can unwind DNA or RNA substrates with a $5^{\prime}->3^{\prime}$ directionality ${ }^{14-16}$. Along with the two canonical RecA ATPase domains of SF1 helicases ${ }^{14,17}$, nsp13 contains three additional domains; an N-terminal zinc-binding domain (ZBD, unique to nidoviral helicases), a stalk, and a 1B domain ${ }^{13,18,19}$. Prior studies established that the nsp13 helicase forms a stable complex with the RTC, and single-particle cryo-electron microscopy (cryo-EM) structures of an nsp13 2 -RTC (the RTC with two nsp13 protomers bound) have been determined ${ }^{20-22}$.

In the nsp132-RTC structure, two protomers of nsp13 (nsp13.1 and nsp13.2; Fig. 1) sit on top of the RTC with each nsp13-ZBD interacting with one of the two Nterminal helical extensions of nsp8 ${ }^{20-22}$. This overall architecture places the nsp13.1 active site directly in the path of the downstream template-RNA (t-RNA). The cryo-EM maps showed that the 5'-single-stranded overhang of the t-RNA (Fig. S1) passed through the nucleic acid binding channel of nsp13.1 23 , but the low resolution of the map due to structural heterogeneity precluded detailed modeling 20 .

The structural analysis of the nsp132-RTC provided new perspectives into the role of the nsp13 helicase in the complex viral replication-transcription program, suggesting that nsp13 may facilitate processive elongation by the RdRp on the highly structured RNA genome ${ }^{24,25}$, but may also generate backtracked RTCs for proofreading, template-switching during sub-genomic RNA transcription, or both ${ }^{20,26}$. How nsp13 directs these diverse processes that regulate RdRp function remains less 
understood. For instance, the structures indicate that nsp13 translocates on the t-RNA strand in the $5^{\prime}->3^{\prime}$ direction ${ }^{16}$, while the RdRp would translocate on the same strand in the opposite direction (Fig. 1B). How can the RdRp rapidly replicate the $\sim 30 \mathrm{~kb}$ viral genome ${ }^{27}$ if it is opposed by the helicase? Also, what is the role of the second nsp13 protomer (nsp13.2), which appears capable of ATPase and translocation/helicase activity but does not appear to be engaged with nucleic acid in the structures ${ }^{20,26}$ ?

Here we describe an extensive structural analysis of a cryo-EM dataset of the nsp13-RTC, combined with molecular dynamics (MD) simulation analysis of the resulting structures. The results yield a cryo-EM map of the nsp132-RTC at a nominal resolution of $2.8 \AA$ (2.1-2.5 $\AA$ in the active site core of the RdRp; Fig. 1). Structural heterogeneity apparent in the nsp13 portions of the map was resolved by classification approaches, revealing four distinct conformational states of the nsp13 subunits. Analysis of these conformational states suggest solutions to the apparent contradictions regarding the role of nsp13 and provides further insight into models for nsp13 function during viral replication/transcription.

\section{RESULTS}

An augmented cryo-EM dataset allows extensive structural analysis of the nsp13RTC. Previously we described a single-particle cryo-EM analysis of a stable SARSCoV-2 nsp13-RTC from a curated set of 88,058 particle images ${ }^{20}$. These particles were classified into three distinct assemblies, nsp13 1 -RTC (4.0 $\AA$ nominal resolution), nsp132RTC $(3.5 \AA)$, and a dimer of nsp13 ${ }_{2}-\mathrm{RTC}\left[\left(\mathrm{nsp} 13_{2}-\mathrm{RTC}\right)_{2} ; 7.9 \AA\right]$. Here we analyzed a much larger dataset (nearly five times as many particles; Fig. S1, Table S1) collected from the same sample preparation. From a consensus refinement (Figs. S1 and S2, map1; Note: Fig. S1 shows the details of the cryo-EM processing pipeline; Fig. S2 is a streamlined cryo-EM processing pipeline that highlights the essential steps), the particles were classified ${ }^{28}$ into the same three assemblies observed previously [nsp131RTC (map2), nsp132-RTC (map3), (nsp13 $2-R T C){ }_{2}$ (map4)] ${ }^{20}$ with a very similar distribution of particles between the three assemblies (Figs. S1 and S2; Tables S1 and $\mathrm{S} 2$ ), confirming the robustness of the classification procedure. We focus primarily on the nsp13 2 -RTC because the bulk of the particles $(72 \%)$ belong to this class and generated the highest resolution map (Figs. S1, S2 and S3; map3; $2.9 \AA$ nominal resolution).

To obtain the best possible consensus cryo-EM map of the entire complex, we generated a series of cryo-EM maps by focused refinement around sub-domains of the nsp131-RTC (map2) and nsp132-RTC (map3) maps and combined these, generating a composite map with a nominal resolution of $2.8 \AA$ (Fig. 1; Figs. S1-S3, map9). Local resolution analysis ${ }^{29}$ suggested that the active site and NiRAN ligand-binding pocket of the RdRp were resolved to between 2.1-2.6 $\AA$ resolution (Fig. S3). This was supported by the excellent quality of the cryo-EM map, where the ADP- $\mathrm{Mg}^{2+}$ bound in the NiRAN domain enzymatic site could be visualized (Fig. S4), and RNA base pairs near the RdRp active site could be identified directly from the cryo-EM density (Fig. S4). Although not as well resolved, the ADP-AlF ${ }_{3}-\mathrm{Mg}^{2+}$ and surrounding residues in the nsp13 active sites could also be modeled (Fig. S4). 
Despite the excellent map quality for most of the RTC (Figs. 1 and S4), features of the composite consensus map (map9) suggested substantial heterogeneity in the nsp13 subunits, particularly in the RecA2 and 1B domains (Fig. S3). Therefore, we generated a mask surrounding the nsp13.1 and nsp13.2 RecA1, RecA2, and 1B domains (of map3; Figs. 2 and S2) and used masked classification with signal subtraction ${ }^{30}$ to identify four distinct conformational states (Figs. 2, S1, S2, and S5; Table S3) with significant differences in the dispositions of the nsp13 subunits, particularly nsp13.1.

The class II structure (Figs. 2 and S5) contains the most particles and the nsp13 subunits are best resolved in this map (map12; Figs. 2, S2 and S5). Compared to the other structures, the nsp13.1 RecA domains of class II (map12) are closed onto each other more than the other structures (Fig. S5) and are thereby engaged most tightly with the RNA (see below). We call this the 'nsp13.1-engaged' structure and use it as a reference to give a general overview of the conformational changes in the other classes.

While each of the classes shows significant changes in both the disposition of each nsp13 subunit as a whole as well as intramolecular domain motions within each nsp13 subunit, each structural class can be characterized by one dominant conformational change in nsp13.1 (compared to the nsp13.1-engaged structure used as a reference):

i) In class I, the nsp13.1 RecA2 domain is rotated open by $21^{\circ}$ with respect to RecA1. Concomitantly, the RNA binding site is empty while occupancy of the nsp13.1 nucleotide-binding site is ambiguous. We therefore call this the 'nsp13.1-apo' structure (Fig. 2).

ii) In class III, the nsp13.1 subunit swivels as a whole by $38^{\circ}$ away from nsp13.2. We call this the 'nsp13.1-swiveled' structure (Fig. 2).

iii) In class IV, the nsp13.1 domain $1 \mathrm{~B}$ is rotated $85^{\circ}$ away from the nsp13.1 RNA binding channel, creating the '1B-open' structure (Fig. 2).

\section{The nsp13.1-engaged conformation grasps the downstream RNA t-strand}

In the nsp13.1-engaged structure, the distance between the center-of-gravity of the two nsp13.1 RecA domains, $27.3 \AA$, is the shortest of the eight nsp13 conformations (Fig. S5). The RecA domains are thus 'closed' and grasp the downstream t-RNA singlestranded 5'-segment emerging from the RdRp active site, giving rise to well-resolved cryo-EM density for the RNA passing through the helicase (Fig. 3A). The RNA is corralled in a tunnel between the two RecA domains and the 1B domain, which is also in a closed conformation (Figs. 2 and $3 \mathrm{~A}$ ). The pattern of purine and pyrimidines in the cryo-EM density is clearly discernable, allowing the unique sequence register of the RNA engaged with the nsp13.1 helicase to be determined (Fig. 3A).

The ordered RNA segment is 7 nucleotides in length (+9 to +15 ; Fig. 3$)$, with the five central nucleotides $(+10$ to +14$)$ completely enclosed within the helicase. The RNA phosphate backbone generally faces the nsp13.1 RecA domains, and the mostly stacked bases face the 1B domain (Fig. 3). As might be expected, the helicase establishes extensive interactions with the RNA phosphate backbone, including several 
polar interactions. Interactions with the RNA bases are mostly van der Waals interactions and not expected to be base-specific (Fig. 3).

\section{The nsp13.1-apo state}

Comparison of the nsp13.1-apo and nsp13.1-engaged structures revealed a striking change in the conformation of the RecA-like ATPase domains of nsp13.1. Superimposition of the $\alpha$-carbons of nsp13.1 RecA1 (residues 235-439) or RecA2 (residues 440-596) alone yielded root-mean-square-deviations (rmsds) of 0.387 and $0.673 \AA$, respectively, indicating the structures of the individual domains are very similar between the two states. However, superimposition via the $\alpha$-carbons of only RecA1 gave an rmsd of $7.05 \AA$ for the RecA2 $\alpha$-carbons, indicating a substantial change in the relative disposition of the two domains. The movement of RecA2 with respect to RecA1 corresponds to an $\sim 21^{\circ}$ rotation about the axis shown in Fig. 4A (also see Video S1), corresponding to an opening of the RecA domains; the nsp13.1 RecA domains of the nsp13.1-apo state are the furthest open of any of the eight nsp13 protomer structures (Fig. S5F).

The consensus nsp132-RTC cryo-EM map (map3; Figs. S1 and S2) contains lowresolution density indicating that the downstream single-stranded 5'-segment of the tRNA occupies the nsp13.1 RNA binding channel (Fig. 1B). Moreover, the t-RNA 5'segment occupying the nsp13.1 RNA binding channel of the nsp13.1-engaged state is well resolved (Fig. 3). By contrast, the nsp13.1-apo cryo-EM density shows that the nsp13.1 RNA-binding path is empty (Fig. 4B). The nsp13.1-apo cryo-EM density also does not support occupancy of ADP-AIF $3-\mathrm{Mg}^{2+}$ in the nucleotide-binding site of nsp13.1, although the low resolution of the map in this region makes this conclusion tentative.

\section{Spontaneous and reversible transition of the nsp13.1 RecA domains between the nsp13.1-engaged and nsp13.1-apo conformations}

To characterize the RecA1-RecA2 interdomain movement and how a bound substrate may influence that movement, we performed MD simulations of free nsp13.1 (i.e., without nsp13.2 or the RTC) under four different substrate-bound conditions (ATPMg ${ }^{2+} / \mathrm{RNA}_{\mathrm{ADPMg}}{ }^{2+} / \mathrm{RNA}, \mathrm{ATPMg}^{2+}$ only, and $\mathrm{ADPMg}^{2+}$ only). For each condition, we ran three independent $5-\mu s$ simulations, all initiated from the nsp13.1-engaged conformation (Figs. 2 and 3).

In simulations of ATPMg ${ }^{2+} / \mathrm{RNA}-$ bound nsp13.1, the RecA2 domain maintained its general orientation with respect to RecA1 throughout the simulations (Fig. 4C). The average rmsd of RecA2 between the initial nsp13.1-engaged cryo-EM structure and the structures from the MD trajectories, aligned on the RecA1, was low ( 2.9 $\AA$; some adjustment of RecA2 from the initial nsp13.1-engaged cryo-EM structure position in these simulations was expected, as the cryo-EM structure was determined using ADP$\mathrm{AIF}_{3} / \mathrm{RNA}$ in place of ATPMg ${ }^{2+} / \mathrm{RNA}$ ). Conformations resembling the nsp13.1-apo structure ( $r m s d<3.5 \AA$ ) were not observed (Fig. 4C, D). 
In simulations of ADPMg ${ }^{2+} / \mathrm{RNA}$-bound nsp13.1, RecA2 rotated away from its initial position in the nsp13.1-engaged conformation, and nsp13.1-apo-like conformations were repeatedly visited throughout the simulations (Figs .4C, D). The $\mathrm{ADPMg}^{2+} / \mathrm{RNA}$-bound nsp13.1-apo-like conformations were metastable, and interconverted with the nsp13.1-engaged conformations. Spontaneous and reversible conversion between the nsp13.1-engaged and nsp13.1-apo conformations was also observed in the simulations of ATPMg ${ }^{2+}$-bound and ADPMg ${ }^{2+}$-bound nsp13.1 (Figs. 4C, D). These results suggest that the presence of both the ATPMg ${ }^{2+}$ and RNA may stabilize the nsp13.1-engaged conformation and that the absence of either substrate may destabilize the nsp13.1-engaged conformation and facilitate the transition to the nsp13.1-apo conformation, consistent with the observations from the cryo-EM analysis.

\section{The '1B-open' conformation of nsp13.1 may explain how the RdRp can synthesize RNA in the presence of nsp13}

In the nsp13.1-engaged state, the downstream single-stranded t-RNA is guided through a deep groove between the RecA1 and RecA2 domains that is completely closed off by the 1B domain (Fig. 5A). Remarkably, in the 1B-open structure, the nsp13.1 1B domain rotates $85^{\circ}$ about the stalk away from the nsp13.1 RNA binding channel, creating an open groove rather than a closed tunnel (Fig. 5B). The cryo-EM density allows modeling of the downstream single-stranded t-RNA emerging from the RdRp active site up to the edge of the open groove proximal to the RdRp, but the RNA density disappears there, indicating that the RNA is not engaged within the active site of the helicase (Fig. 5B).

In the 1B-open conformation, the nsp13.11B domain appears to be trapped open by the presence of nsp13.2 (Fig. 5B), with the transition from the 1B-open to the 1B-closed conformation blocked by nsp13.2. Consistent with this, we analyzed the nsp13 conformational states in the nsp131-RTC (nsp13.2 absent) by masked classification with signal subtraction around the RecA1, RecA2 and 1B domains of the single nsp13 (Fig. S1) but the 1B-open nsp13 conformation was not observed. We propose that the 1B-open conformation of the nsp13.1 1B domain is trapped by the presence of nsp13.2.

We note that in the (nsp132-RTC) 2 dimer (Figs. S1-S3), the nsp13 protomers corresponding to nsp13.1 are also in the '1B-open' state, as was observed by Yan et al. 31. Since the dimer only comprises $8 \%$ of our particle dataset (Table S1) while the nsp132-RTC complex comprises $72 \%$ of the particles, we have focused our attention on the latter complex. We observe that the (nsp132-RTC) 2 dimer forms in the absence of additional factors such as nsp10-14 ${ }^{20}$, in contrast to what's reported in Yan et al. ${ }^{31}$.

Yan et al. ${ }^{31}$ observed the 1B-open state of nsp13.1 (labeled nsp13-2 in their nomenclature) in their (dimer) dCap(0)-RTC structure, curiously assigned as a backtracking-competent state. This is at odds with: i) observations that nsp13.1 in the 1B-open conformation does not engage RNA in its RNA-binding groove [Fig. 5B; also observed by Yan et el. ${ }^{31}$ ] and so would be incompetent for RNA translocation, and ii) the finding that nsp13 stimulated SARS-CoV-2 RTC backtracking ${ }^{26}$. 


\section{Spontaneous transition of the nsp13.1 1B domain from the 1B-open to 1B-closed} conformations

The conformations of the nsp13.1 1B domain in the nsp13.1-engaged and nsp13.1-apo structures are related by a $\sim 10^{\circ}$ rotation around the nsp13-stalk, but the $1 \mathrm{~B}$ domains are closed on the nsp13-RecA domains in both structures. We refer to these collectively as '1B-closed' states (Fig. 2). These conformations have also been observed in crystal structures of isolated nsp13 as well as some other SF1-like helicases ${ }^{32}$. The conformation of the 1B domain in the 1B-open cryo-EM structure, in which the domain is rotated $\sim 85^{\circ}$ compared to the $1 \mathrm{~B}$-closed conformations, was only seen in nsp 13.1 when it was paired with nsp13.2 in the RTC, suggesting that this conformation may not be stable in isolated nsp13. To test this hypothesis, we performed five independent $25-\mu \mathrm{s}$ simulations on isolated (free) nsp13 (with $\mathrm{ADPMg}^{2+}$ ), initiated from the 1B-open conformation (Fig. 2).

In three out of the five simulations, the $1 \mathrm{~B}$ domain underwent a $\sim 90^{\circ}$ rotation from the starting $1 \mathrm{~B}$-open conformation around the stalk toward the RNA-binding groove to a $1 \mathrm{~B}$-closed conformation (Fig. $6 \mathrm{~A}$ ). These $\sim 90^{\circ}$ rotated $1 \mathrm{~B}$ domain conformations closely resemble the disposition of the $1 \mathrm{~B}$ domain in the nsp13.1-apo structure. The 1B domain rmsd between the simulation-generated structures from the last $2 \mu \mathrm{s}$ of the three trajectories and the 1B domain in the nsp13.1-apo cryo-EM structure (aligned on the RecA1 domain) was, on average, $\sim 3.6 \AA$. We also observed that a small portion $(<5 \%)$ of these $1 \mathrm{~B}$-closed structures were more similar to the 1B domain of the nsp13.1engaged conformation ( $r m s d<3.5 \AA$ ). These nsp13.1-engaged-like 1B conformations were short-lived, and once visited they quickly transitioned to the nsp13.1-apo conformation, presumably because the nsp13.1-engaged conformation was captured in the presence of RNA, which was not included in our simulations.

We next asked whether or not the 1B domain in a 1B-closed state may spontaneously transition to the 1B-open state. In each of the three simulations in which we observed a transition of the $1 \mathrm{~B}$ domain from the 1B-open to a 1B-closed conformation, the $1 \mathrm{~B}$ domain remained in the 1B-closed conformation through the end of the 25- $\mu$ s simulation; a 1B-closed-to-open transition was not observed. We performed an additional five independent $25-\mu$ s simulations of the isolated (free) nsp13.1, initiated from the 1B-closed conformation (of the nsp13.1-engaged structure). We did not observe any transition events to the 1B-open conformation over the aggregated $125 \mu \mathrm{s}$ simulation time. Instead, the 1B domain maintained its 1B-closed orientation in the initial structure, with some minor wobbling back and forth between the 1B-closed conformations of the nsp13.1-engaged and nsp13.1-apo structures (Fig. 6B).

Aligning the nsp13.1 simulation structures in the 1B-open-to-closed transition pathways with the nsp13.1 of the 1B-open cryo-EM structure showed that, on average, $\sim 40 \%(53 \%, 22 \%$, and $45 \%$ in the three simulations) of these $1 \mathrm{~B}$ domain intermediate conformations clashed with nsp13.2, suggesting that the 1B-open-to-closed transition might be blocked by nsp13.2 (Fig. S6). Here we envisage that 1B domain transitions are facilitated by entry into the 'swiveled' state. The swiveled structure is characterized by one dominant conformational change; compared to the nsp13.1-engaged structure, the 
nsp13.1 protomer as a whole swivels with respect to the rest of the RTC by $38^{\circ}$, repositioning nsp13.1 with respect to nsp13.2 (Fig. 6C).

There are some clashes between nsp13.1 and nsp13.2 when the simulationgenerated structures are aligned to the swiveled cryo-EM structure, but to a much lesser extent $(9 \%, 0 \%$, and $2 \%$ in the three simulations; Fig. S6). This observation is consistent with the notion that the swiveled structure may be an intermediate state that facilitates the transition between the 1B-open and 1B-closed conformations.

In summary, our simulations suggest that the conformation of the $1 \mathrm{~B}$ domain in the 1B-open structure may only be transiently stable on its own, transitioning spontaneously into the 1B-closed conformations of the nsp13.1-apo and nsp13.1engaged structures. Such transitions may be blocked by the presence of nsp13.2 in the 1B-open nsp132-RTC. We did not observe transitions from the 1B-closed conformations to the 1B-open conformation, and we speculate that in the presence of RNA in the nsp13.1 RNA-binding groove (Fig. 3), nsp13.1 may be further stabilized in the closed 1B domain conformation.

\section{Nsp13 conformations in nsp132-backtracked complexes}

In the nsp13.1-engaged state (Fig. 2), the RdRp translocates in the $3^{\prime}->5^{\prime}$ direction on the t-RNA while nsp13.1 grasps the single-stranded t-RNA ahead of the RdRp (Fig. 3) and translocates in the $5^{\prime}->3^{\prime}$ direction (Fig. 1B). We proposed that events at the RdRp active site that would delay or stall p-RNA chain elongation (such as misincorporation or incorporation of nucleotide analogs) could allow the nsp13.1 translocation activity to push the RdRp backward on the t-RNA ${ }^{20}$. In this process, termed backtracking, the complex moves in the $5^{\prime}->3^{\prime}$ direction on the t-RNA accompanied by reverse-threading of the p-RNA through the complex, generating a single-stranded p-RNA 3 '-fragment. In support of this hypothesis, structural and functional studies showed that the SARS-CoV2 RdRp can backtrack, that the resulting single-stranded p-RNA 3'-fragment extrudes out the RdRp NTP-entry tunnel, and that backtracking is stimulated by nsp $13^{26}$.

To compare the conformational states of the nsp13 protomers in the nsp132BTCs (backtracked complexes) with the nsp132-RTCs, we used the same masked classification with signal subtraction protocol (Fig. S2) to classify the nsp132-BTC particles into four conformational states (Fig. S7). Structural models were built and rigidbody refined into the cryo-EM densities for each class except for nsp132-BTC-class2 (13\% of the particles), which had very poor cryo-EM density for nsp13.1. To compare these structural models with the nsp13 2 -RTC structures, we aligned the models for each nsp132-BTC model with the nsp13.1-engaged state by superimposing $\alpha$-carbons of nsp12, yielding rmsds $<0.213 \AA$. We then calculated rmsds for $\alpha$-carbons of nsp13.1 and nsp13.2. Both nsp132-BTC-class1 and nsp132-BTC-class4 aligned well with the nsp13.1-engaged nsp132-RTC state (Table S4) and both also had strong density for the downstream t-RNA engaged with nsp13.1 (Fig. S7). Therefore, we classify both of these structures as nsp13.1-engaged-BTCs. The nsp132-BTC-class3 structure had an open 1B domain of nsp13.1 and clearly aligned with the 1B-open-RTC structure (Table S4). Thus, in contrast to the nsp132-RTC structures, which were equally divided between the nsp13.1-engaged and 1B-open states (33\% each), the nsp132-BTC structures were 
heavily skewed towards the nsp13.1-engaged state (72\%) vs. the $1 \mathrm{~B}$-open state (15\%; Fig. S7).

\section{Discussion}

In this work, we observed distinct conformational states of the nsp13 protomers within the SARS-CoV-2 nsp132-RTC, providing functional insights into nsp13 and its complex with the RTC (see Video S2). Like other helicases, nsp13 is a molecular motor that translocates along single-stranded nucleic acid, unwinding structural elements in its path (Mickolajczyk et al., 2020). This process is driven by conformational changes within nsp13 resulting from NTP hydrolysis.

The conformational transition from the nsp13.1-engaged to the nsp13.1-apo structures, observed both by our cryo-EM (Fig. 4A) and MD (Figs. 4C, D) analyses, corresponds to an $\sim 21^{\circ}$ rotation of the RecA2 domain with respect to RecA1, opening the gap between the two domains (Fig. 4A; Video S1). The nsp13.1-engaged structure is engaged with the substrate RNA and is trapped in an 'on-pathway' conformation of the nucleotide hydrolysis cycle by the non-hydrolyzable ATP analog ADP-AIF 3 . While the nsp13.1-apo structure, being devoid of RNA, is not 'on-pathway' per se, the $21^{\circ}$ opening of the RecA2 domain from the nsp13.1-engaged to nsp13.1-apo conformations matches the disposition of the RecA2 domains in other SF1 helicases, such as human Upf1, a structural homolog of nsp13 ${ }^{13,23}$. The disposition of the RecA domains of Upf1 with $\mathrm{ADP}_{-}-\mathrm{AlF}_{3}$ and RNA substrate [PDB 2XZO] ${ }^{33}$ matches the nsp13.1-engaged structure. On the other hand, the RecA domains in a structure of Upf1 with ADP (so likely on-pathway) are opened by a $24^{\circ}$ rotation about the same axis as the $21^{\circ}$ opening of the nsp13.1-apo RecA domains [PDB 2GK6] ${ }^{34}$. We thus infer that the nsp13.1-apo conformation reports on an on-pathway conformation of the RecA domains, such as in the ADP-Mg ${ }^{2+} / \mathrm{RNA}-$ bound state of the translocation cycle (Figs. 4C, D). Due to the opening of the nsp13.1 RecA domains, the center-of-gravity of RecA2 shifts roughly parallel with the RNA backbone by $3.4 \AA$, corresponding to the rise between stacked RNA bases. This observation is suggestive of an 'inchworm' model for translocation (Video S1), as proposed for related SF1 helicase translocation on single-stranded nucleic acids ${ }^{14,17,35-39}$.

Prior structural analysis of the nsp132-RTC identified that the nsp13.1 helicase and the RdRp translocate on the t-RNA with opposing polarities (Chen et al., 2020). In circumstances where RdRp elongation of the p-RNA is hindered (such as in the event of a misincorporation at the p-RNA 3'-end), nsp13.1 translocation activity could backtrack the RdRp ${ }^{20}$, as shown by follow-up structural and biochemical analyses ${ }^{26}$. The opposing polarities of the RdRp and nsp13 translocation activities (Fig. 1B) presented a conundrum that was not addressed by these previous studies; how is it possible for the RdRp to rapidly and efficiently synthesize RNA if it is constantly being opposed by nsp13? Moreover, the predominant complex present in the nsp13-RTC samples is the nsp132-RTC complex (Table S1), but only nsp13.1 was seen to engage with the t-RNA; what is the role of nsp13.2, the second copy of nsp13 in the nsp132-RTC? The work herein suggests answers to both questions. 
Maximum likelihood classification approaches revealed four distinct conformations of the nsp13 protomers in the nsp132-RTC (Figs. 2, 7; Videos S1, S2). The nsp13.1-engaged state resolves nsp13.1 clamped onto the single-stranded downstream t-RNA, providing an atomic view of nsp13 engaged with the singlestranded RNA (Fig. 3). The single-stranded t-RNA threads through a tunnel formed by a deep groove between the $\operatorname{Rec} A 1$ and $\operatorname{Rec} A 2$ domains and further enclosed by the $1 \mathrm{~B}$ domain (Fig. 5A).

By contrast, the 1B-open state shows nsp13.1 adopting a conformation in which the $1 \mathrm{~B}$ domain is rotated open $\sim 85^{\circ}$ about the stalk domain, leaving an open RNA binding groove (Fig. 5B). In this state, the single-stranded downstream t-RNA does not engage with the helicase. Thus, this represents an inactive state of the helicase that would be unable to translocate on the RNA.

\section{Our structural analysis combined with MD simulations confirmed that the} conformation of the nsp13.1 1B domain in the 1B-open structure is not stable on its own but is sterically trapped by the presence of nsp13.2, which blocks the conformational change required for $1 \mathrm{~B}$ domain closure (Fig. 6A). These results suggest that the $1 \mathrm{~B}-$ open state represents a rapidly elongating state of the nsp132-RTC, where the downstream single-stranded template RNA feeds into the RdRp active site without engaging with nsp13.1. Nsp13.2 may trap the 1B-open (inactive) state of nsp13.1, allowing RdRp elongation to proceed without opposition from the nsp13.1 helicase (Fig. 7). Finally, swiveling of nsp13.1 in the swiveled state allows space for the 1B-open to $1 \mathrm{~B}$-closed transition (Fig. 6C), suggesting that the swiveled state represents a transition state between the open and closed states of the 1B domain (Fig. 7). We note that the presence of nsp13.2 in the nsp13.1-engaged state would also block the 1Bclosed to 1B-open transition, suggesting how nsp13.2 can enhance the helicase activity of nsp13.1 21

Thus, our results suggest a mechanism for the nsp132-RTC to turn backtracking on and off; switching between rapid RNA synthesis (1B-open state; elongating RdRp; Fig. 7) and backtracking (nsp13.1-engaged, backtracking RdRp; Fig. 7). In our analysis of the conformational states of the nsp132-RTC, the particles were equally divided between the nsp13.1-engaged (backtracking on) and 1B-open (backtracking off) states (Figs. S2, S7). Remarkably, an identical analysis of the backtracked nsp132-BTC 26 revealed a strikingly different distribution of particles in which the nsp13.1-engaged (backtracking on) state was heavily favored (Fig. S7). This raises the possibility that the conformational switch that turns backtracking on and off is allosterically controlled.

In MD simulations exploring the dynamics of the p-RNA 3'-nucleotide of a pretranslocated RTC, a mismatched p-RNA 3'-nucleotide frayed from the t-RNA towards and into the NTP-entry tunnel (which also serves as the backtracking tunnel; Video S1), while a p-RNA 3'-nucleotide engaged in a Watson-Crick base pair with the t-RNA did not ${ }^{26}$. We thus suggest that misincorporation by the RdRp leads to fraying of the $p-R N A$ 3'-nucleotide into the NTP-entry tunnel, which may allosterically signal the rapidly elongating 1B-open state to switch to the backtracking nsp13.1-engaged state (Fig. 7). This facilitates a possible proofreading mechanism since backtracking would extrude the mismatched p-RNA 3'-nucleotide out of the NTP-entry tunnel (Fig. 7) ${ }^{26}$, allowing the nsp10/nsp14 3'-exonuclease proofreading activity to access and degrade the 
mismatched p-RNA 3'-nucleotide ${ }^{40-42}$. The nsp14-mediated proofreading activity is crucial for the virus to avoid mutation catastrophe while replicating its $\sim 30$ kb genome ${ }^{41}$, and is also an important determinant of SARS-CoV-2 susceptibility to many anti-viral nucleotide analogs ${ }^{43}$.

Acknowledgments. We thank M. Ebrahim and L. Urnavicius at The Rockefeller University Evelyn Gruss Lipper Cryo-electron Microscopy Resource Center and H. Kuang at the New York Structural Biology Center (NYSBC) for help with cryo-EM data collection, and R. Landick, T. Appleby, and members of the Darst/Campbell laboratory for helpful discussions, and M. Grasso, P.M.M. Shelton, T. M. Kapoor, P.D.B. Olinares, and B.T. Chait (The Rockefeller University) for helpful discussions and initial sample characterizations and analyses. Some of the work reported here was conducted at the Simons Electron Microscopy Center (SEMC) and the National Resource for Automated Molecular Microscopy (NRAMM) and National Center for CryoEM Access and Training (NCCAT) located at the NYSBC, supported by grants from the NIH National Institute of General Medical Sciences (P41 GM103310), NYSTAR, the Simons Foundation (SF349247), the NIH Common Fund Transformative High Resolution Cryo-Electron Microscopy program (U24 GM129539) and NY State Assembly Majority. This work was supported by the Pels Family Center for Biochemistry and Structural Biology (The Rockefeller University), and NIH grants R01 GM114450 (E.A.C.), R35 GM118130 (S.A.D.), and R01 Al161278 (E.A.C./S.A.D).

Author contributions. Conceptualization; J.C., Q.W., B.M., J.P., E.A.C., D.E.S., S.A.D.; Cloning, protein purification, biochemistry; J.C., B. M., E.L.; Cryo-EM specimen preparation; J.C., B.M., E.L.; Cryo-EM data collection and processing: J.C., K.M., E.T.E.; Model building and structural analysis: J.C., B.M., E.A.C., S.A.D.; Molecular dynamics simulation and analysis; Q.W., Y.P.; Funding acquisition and supervision: E.A.C., D.E.S., S.A.D.' Manuscript first draft: Q.W., B.M., J.P., E.A.C., D.E.S., S.A.D.; All authors contributed to finalizing the written manuscript.

Competing interests. The authors declare there are no competing interests. 


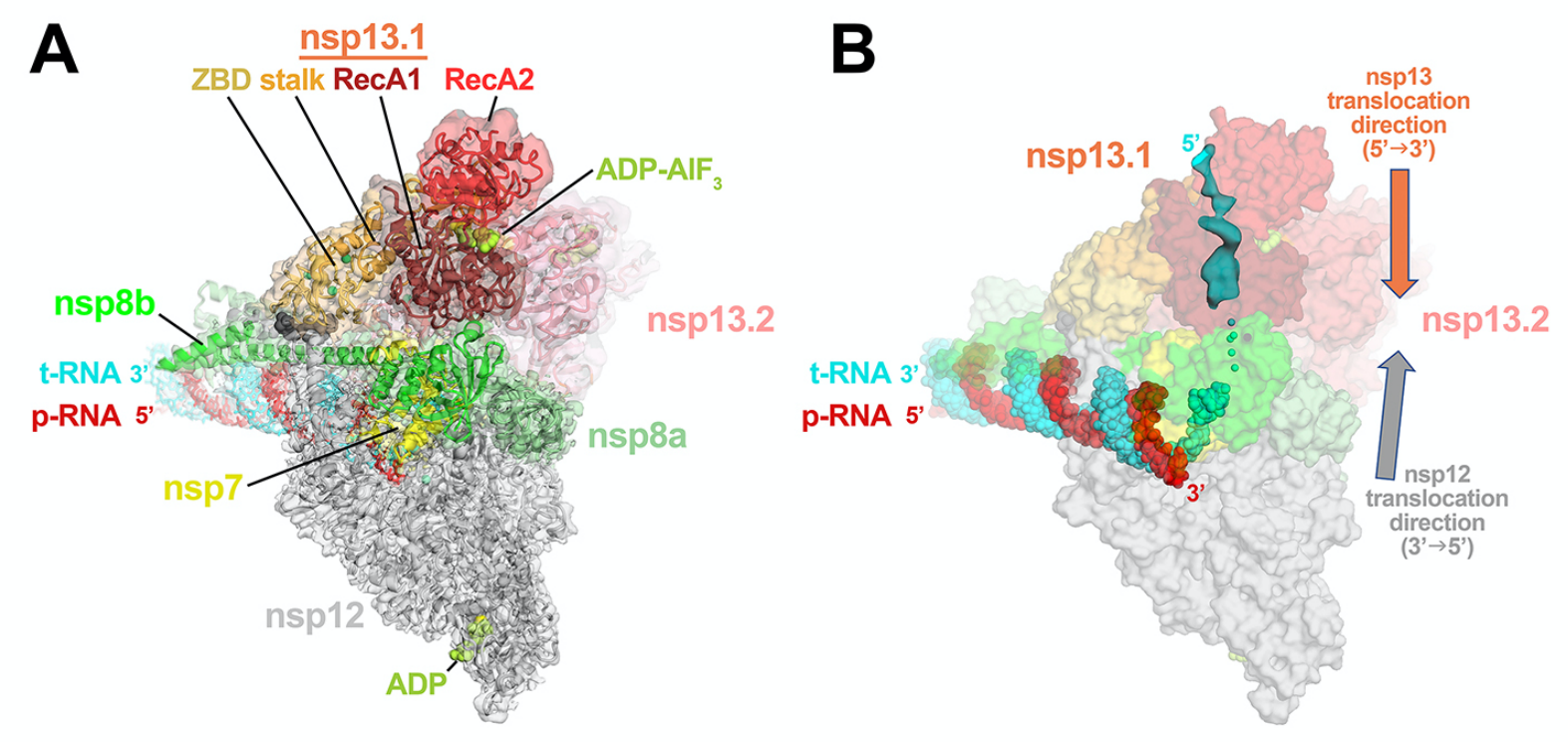

Chen et al., Figure 1

449 Fig. 1 | Consensus cryo-EM structure of an nsp132-RTC.

450 A. Overall architecture of the consensus nsp132-RTC. Shown is the transparent cryo451 EM density (map3, local-resolution filtered) with the nsp13 2 -RTC model superimposed.

452 B. The consensus nsp132-RTC structure is shown; RNA is shown as atomic spheres, 453 proteins are shown as transparent molecular surfaces. A low-pass filtered (6 Å) cryo-EM 454 difference density reveals the path of the downstream t-RNA 5'-segment through the 455 RNA binding groove of nsp13.1 (cyan surface).

456 See also Figs. S1-S4. 


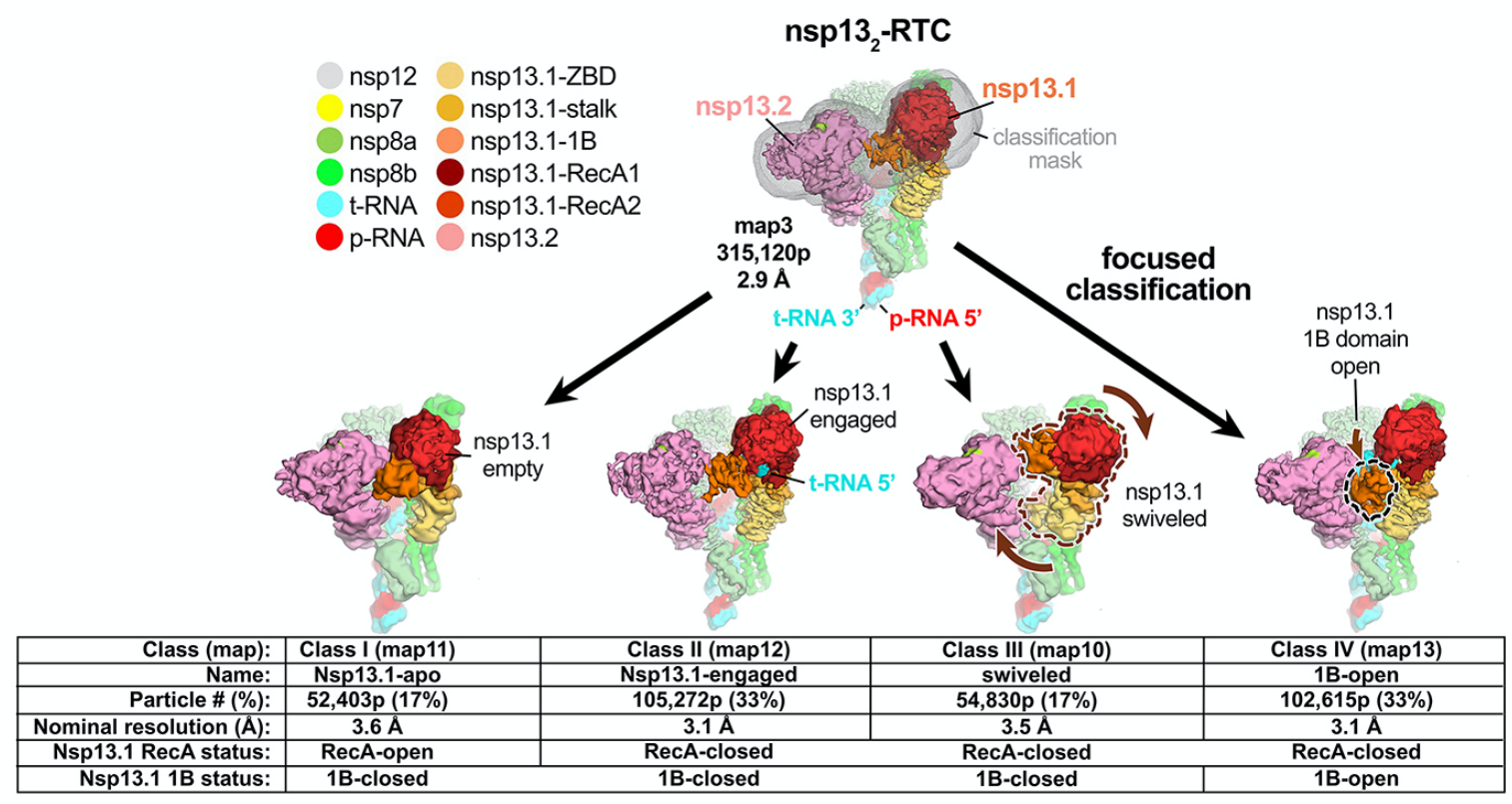

Chen et al., Figure 2

Fig. 2 | Four conformational states of the nsp132-RTC.

461 (top) Cryo-EM density (map3, local-resolution filtered) colored according to the code on the left. A mask was constructed surrounding the nsp13.1 and nsp13.2 1B, RecA1, and RecA2 domains (grey mesh). The 315,120 particles were divided into four distinct structures (class I, II, III, and IV) by focused classification inside the mask, followed by further refinement (Figs. S1, S5). Class II contained the most particles, and the nsp13.1 RecA domains were completely closed (Fig. S5), entrapping the 5'-t-RNA segment in a groove between the two RecA domains and the 1B domain (Fig. 3). Therefore, class II (nsp13.1-engaged) was used as a reference for comparison of the other structures. Each class was characterized by one dominant conformational change: class I) nsp13.1-apo, the RecA domains were completely open (Fig. S5) and devoid of RNA (Fig. 4), class III) swiveled, the nsp13.1 protomer as a whole was rotated $38^{\circ}$ as shown (Fig. 6), class IV) 1B-open, the nsp13.11B domain was rotated open by $85^{\circ}$

473 (Fig. 5).

474 Also see Fig. S5 and Videos S1 and S2. 

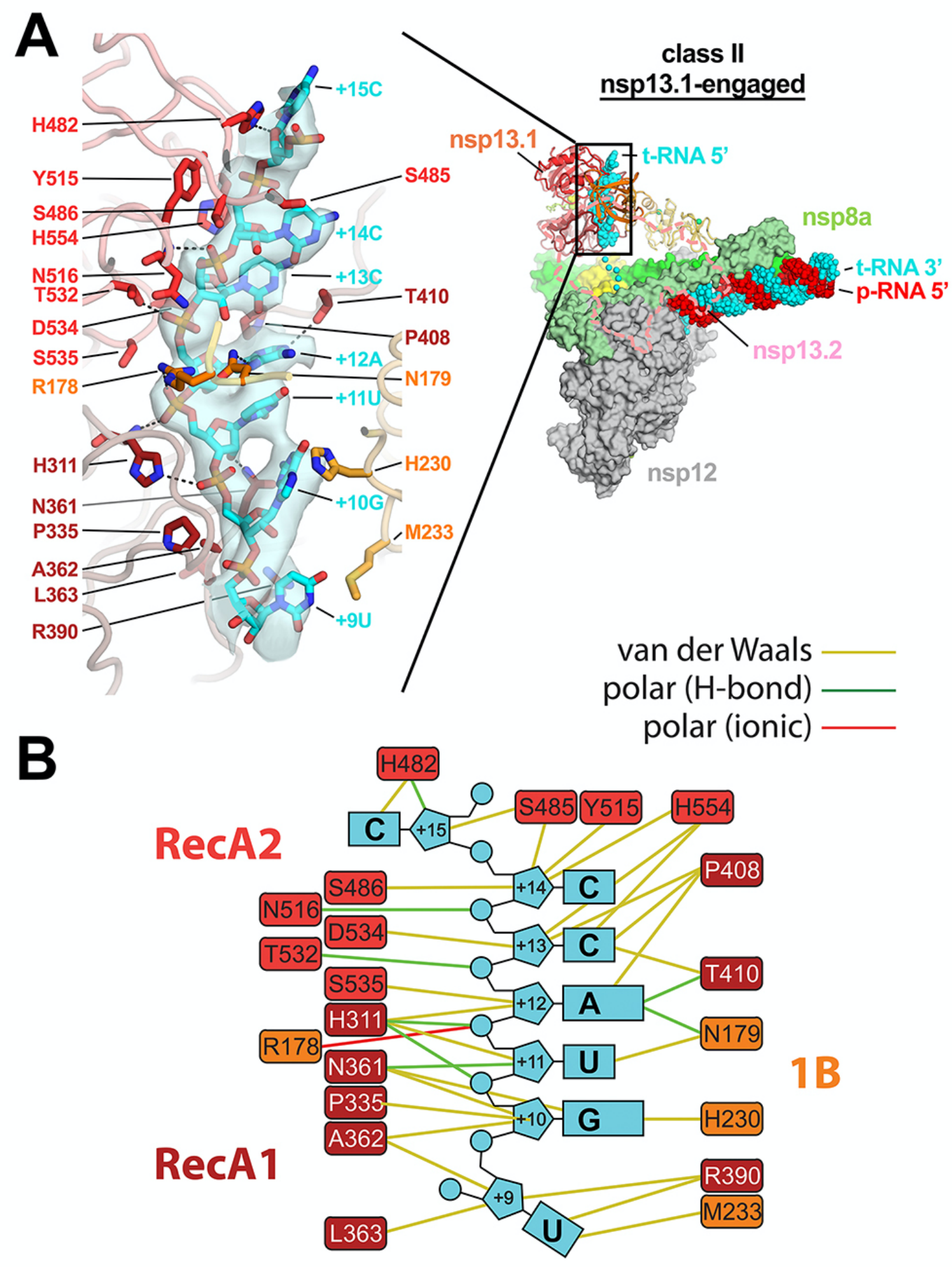
479 Fig. 3 | In class II (nsp13.1-engaged), the nsp13.1 RecA domains and 1B domain 480 clamp onto the 5 '-single-stranded t-RNA.

481 A. (right) Overall view of the nsp13.1-engaged structure. Proteins are shown as

482 molecular surfaces except nsp13.1 is shown as a backbone ribbon, and nsp13.2 is

483 removed and shown only as a dashed outlline. The RNA is shown as atomic spheres.

484 The boxed region is magnified on the left.

485 (left) Nsp13.1 is shown as a backbone worm but with side chains that interact with the t486 RNA shown. Cryo-EM density for the downstream 5'-t-RNA segment is shown

487 (transparent blue surface) with the t-RNA model superimposed. The pattern of

488 purines/pyrimidines in the RNA density was clear and unique, allowing the identification 489 of the sequence register for the nsp13.1-bound RNA.

490 B. Schematic illustrating nsp13.1-RNA interactions. 
bioRxiv preprint doi: https://doi.org/10.1101/2021.11.10.468168; this version posted November 12, 2021. The copyright holder for this preprint (which was not certified by peer review) is the author/funder, who has granted bioRxiv a license to display the preprint in perpetuity. It is made available under aCC-BY-NC-ND 4.0 International license.
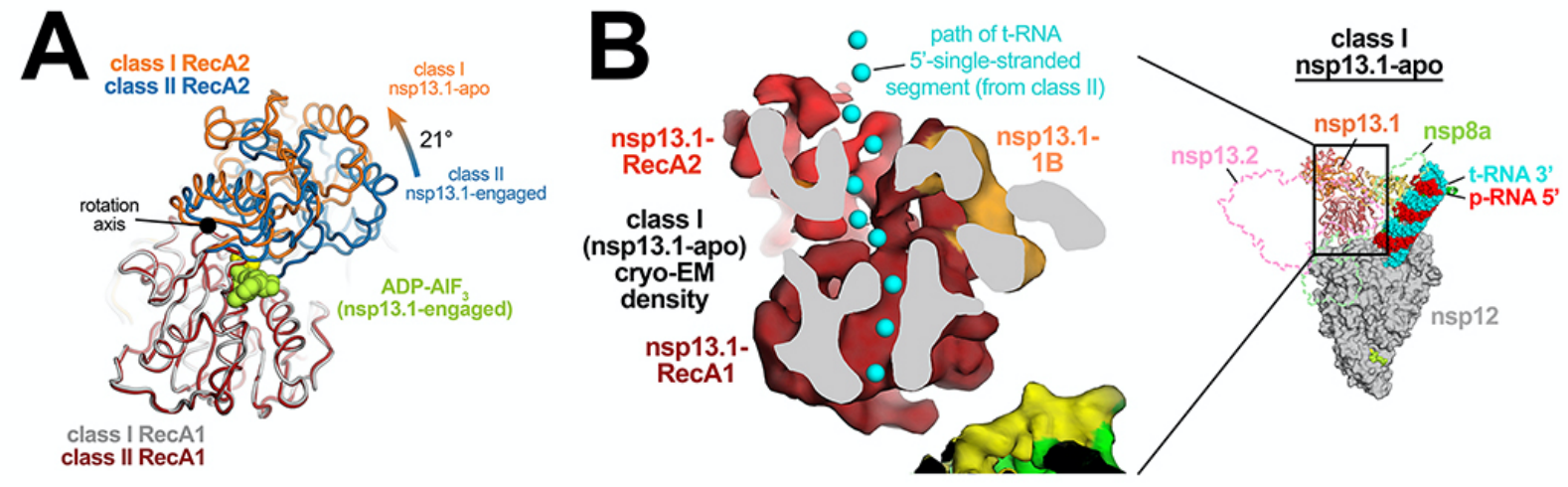

\section{C}

apo engaged
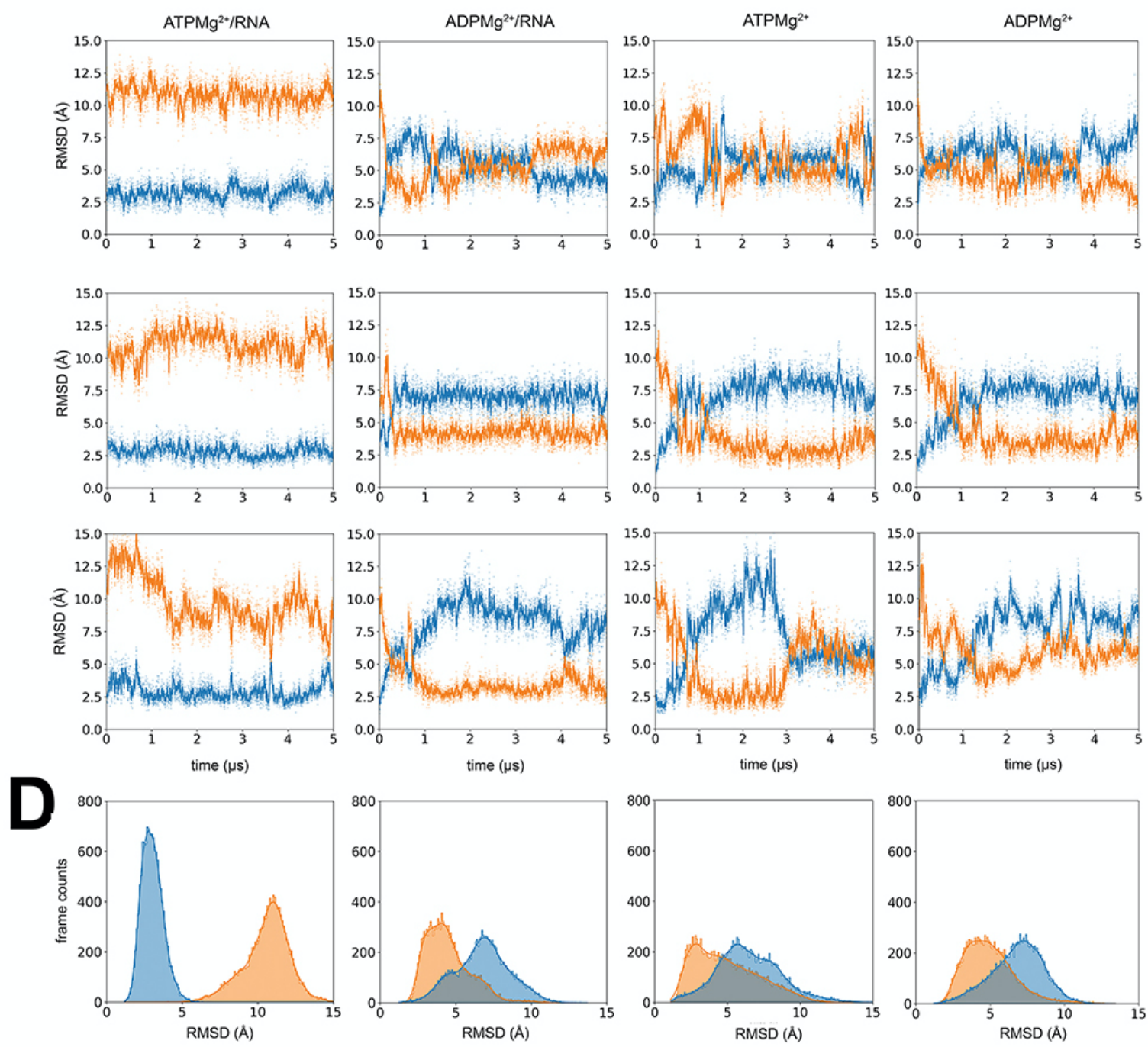

\section{Chen et al., Figure 4}


495 Fig. 4 | In nsp13.1-apo, the RecA domains are open and devoid of RNA density.

496 A. The RecA1 domains of class I (nsp13.1-apo) and class II (nsp13.1-engaged) were 497 superimposed ( $r m s d$ of $0.387 \AA$ over $205 \alpha$-carbons), revealing that the RecA2 domain 498 of nsp13.1-apo was rotated open by $21^{\circ}$ about the rotation axis shown. The ADP-AlF $3^{-}$ $499 \mathrm{Mg}^{2+}$ from the nsp13.1-engaged structure is shown as limon atomic spheres.

500 B. (right) Overall view of the nsp13.1-apo structure. Proteins are shown as molecular 501 surfaces except nsp13.1, which is shown as a backbone ribbon, and nsp13.2, which is 502 removed and shown only as a dashed outline. The RNA is shown as atomic spheres. 503 The boxed region is magnified on the left.

504 (left) Cryo-EM density of nsp13.1-apo reveals that the RNA path is empty (the RNA path 505 from the nsp13.1-engaged structure is denoted by cyan spheres).

506 C. Three independent simulations of nsp13.1 bound to ATPMg ${ }^{2+} / \mathrm{RNA}$ ADPMg ${ }^{2+} / \mathrm{RNA}^{2}$, 507 ATP $\mathrm{Mg}^{2+}$, and $\mathrm{ADPMg}^{2+}$. Values of rmsd plotted represent the heavy-atom rmsd of the 508 RecA2 lobe (after alignment on the RecA1 lobe) with respect to nsp13.1-engaged (blue) 509 and nsp13.1-apo (orange) cryo-EM structures.

510 D. The rmsd histograms represent aggregate values across all three replicates shown 511 in (C).

512 Also see Video S1. 


\section{A Class II $\quad$ B Class IV (nsp13.1-engaged) $\quad$ (1B-open)}
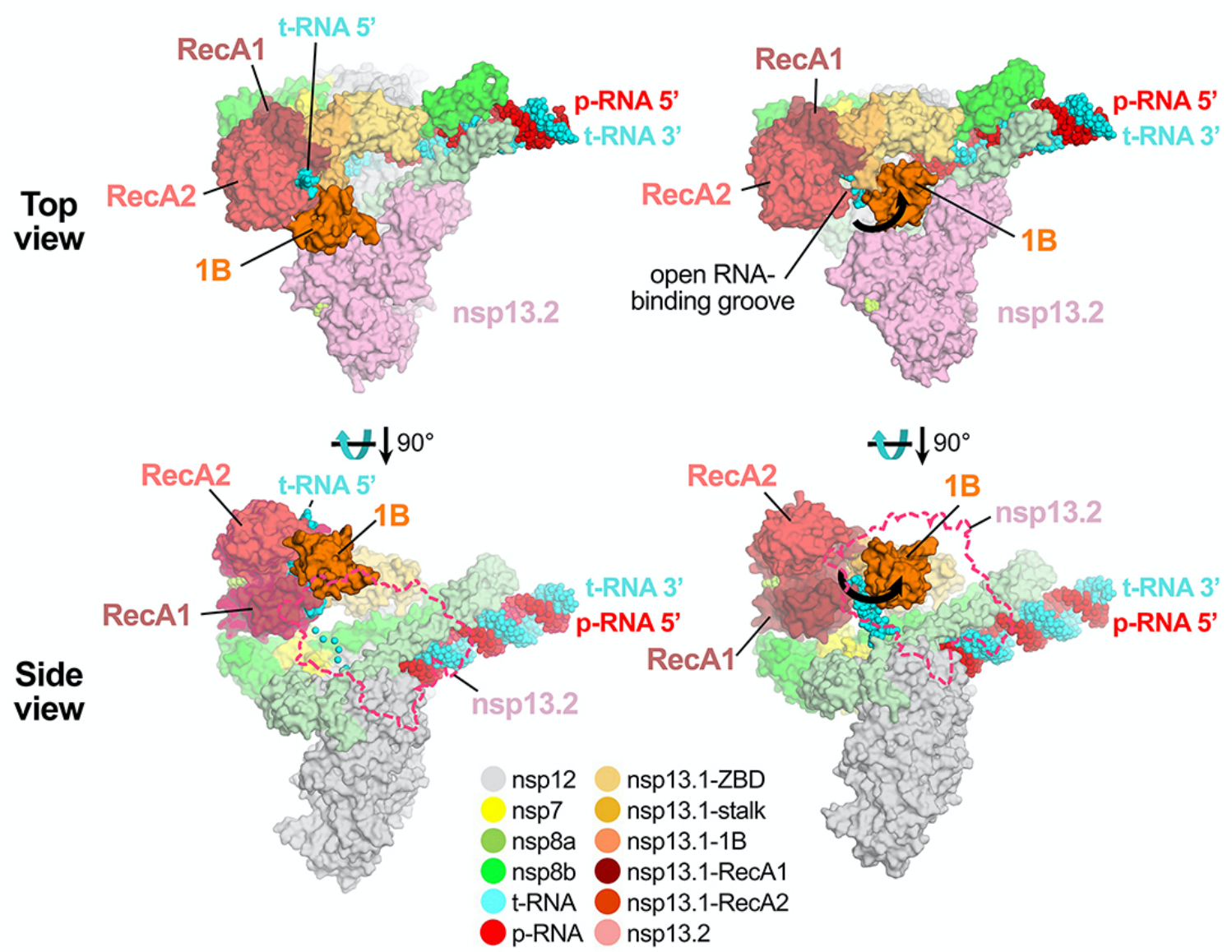

\section{Chen et al., Figure 5}

\section{Fig. 5|1B-open structure.}

517 Comparison of nsp13.1-engaged (A) and 1B-open (B) structures. Two views are shown, 518 a top view (top) and a side view (bottom). In the top view, the proteins are shown as 519 molecular surfaces and color-coded according to the key at the bottom. In the side view, 520 nsp13.2 is shown only as a dashed outline. The RNA is shown as atomic spheres. In 521 the 1B-open structure (B), the nsp13.11B domain is rotated open by $85^{\circ}$ (represented 522 by thick black arrows). The 5'-t-RNA emerging from the RdRp active site approaches 523 the nsp13.1 RNA binding groove but does not enter it.

\section{Also see Video S2.}



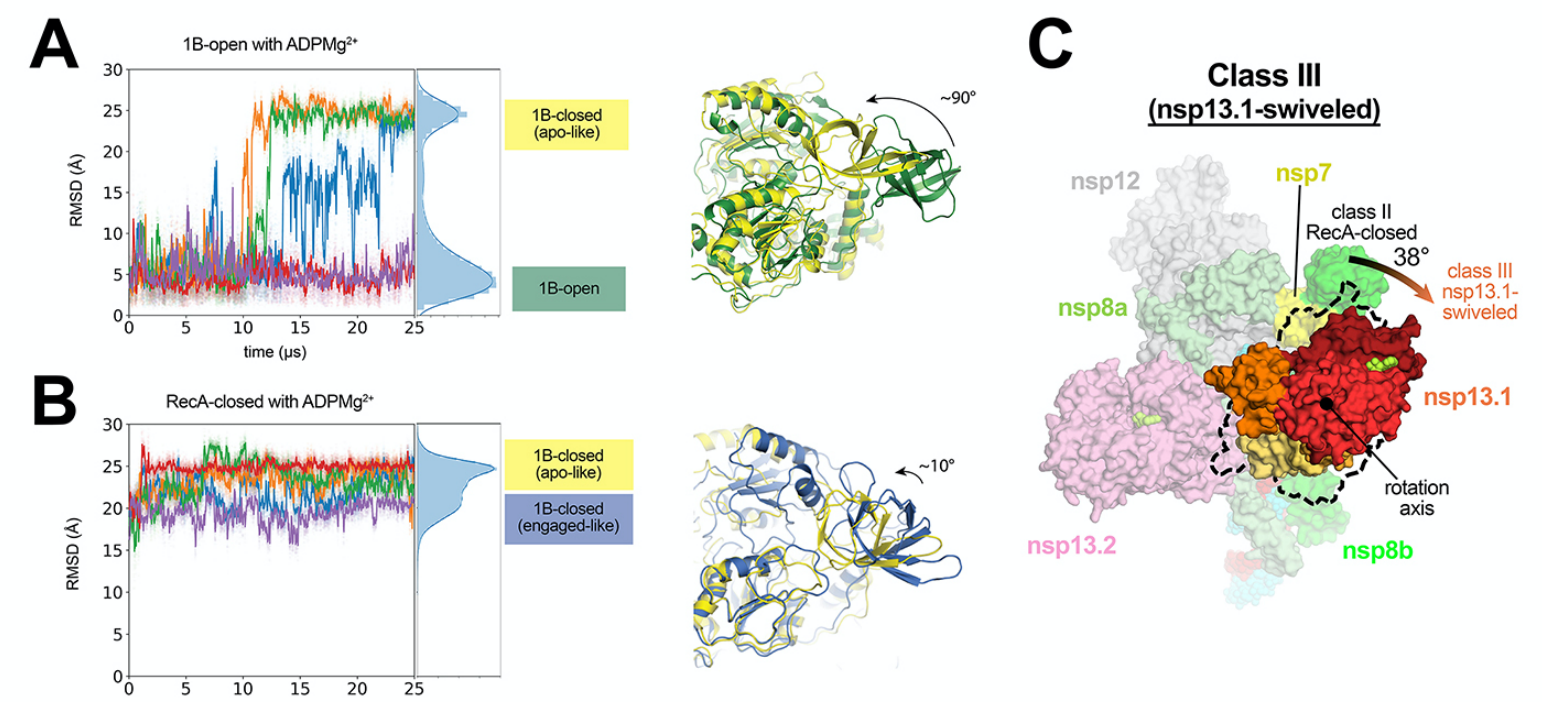

B
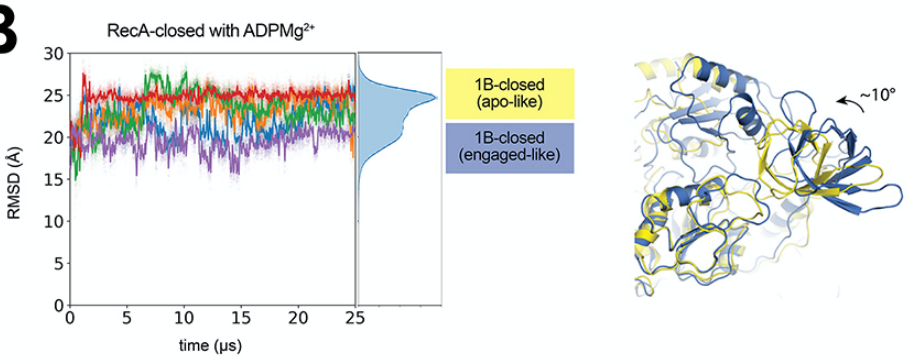

528 Fig. 6 | In the nsp13.1-swiveled structure, the entire nsp13.1 promoter is rotated.

529 A. Front view of the nsp13.1-swiveled structure, highlighting nsp13.1. The position of 530 the nsp13.1 promoter in the nsp13.1-engaged structure is illustrated by the dashed black outline. The nsp13.1 promoter of the nsp13.1-swiveled structure is rotated by $38^{\circ}$ as shown.

B. Five independent simulations of $\mathrm{ADPMg}^{2+}$-bound nsp13.1, starting from the 1B-open cryo-EM structure. Values plotted represent the heavy-atom rmsd of the $1 \mathrm{~B}$ domain (nsp13 residues 150-228) compared to the 1B domain in the 1B-open cryo-EM structure (aligned on the RecA1 domain). The rmsd histograms on the right represent aggregate values across all five simulations. Representative structures of the two major conformations from the rmsd histogram from simulations are shown (right). engaged state. Values plotted represent the heavy-atom rmsd of the 1B domain compared to the 1B domain in the 1B-open cryo-EM state (aligned on the RecA1 domain). The rmsd histograms on the far right represent aggregate values across all five simulations. Representative structures of the two major conformations from the

545 See also Fig. S6. 


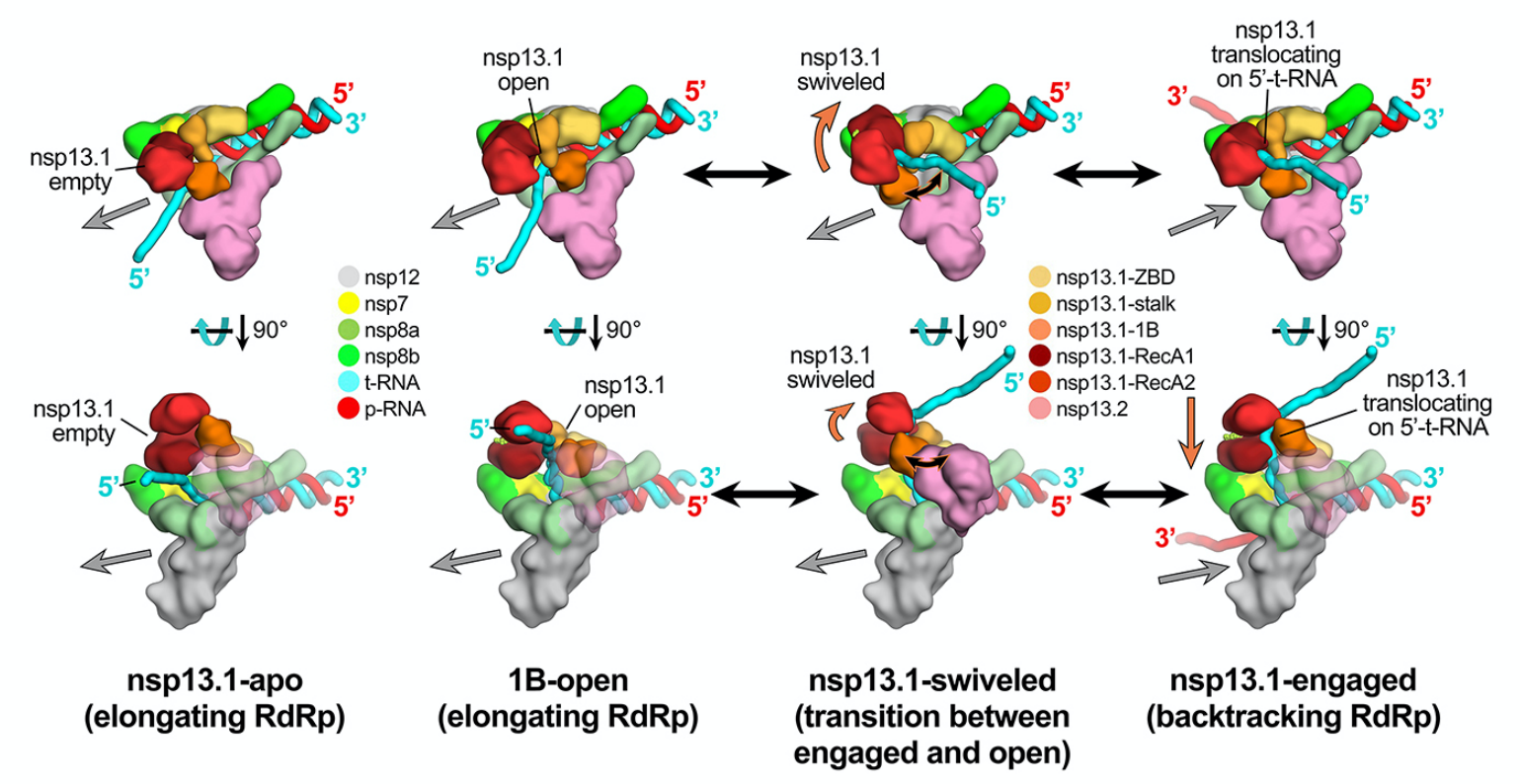

Fig. 7. Schematic model for RTC elongation (1B-open) vs. backtracking (nsp13.1engaged) states.

549 Top views (top row) and side views (bottom row) of each structural class.

550 Nsp13.1-apo (17\%): The nsp13.1 RecA domains are open, consistent with the absence 551 of nucleotide. The nsp13.1 is therefore not engaged with the downstream 5'-t-RNA and the RdRp can freely translocate on the t-RNA with concurrent elongation of the p-RNA

553 (gray arrow pointing downstream).

554 1B-open (33\%): The nsp13.1 1B domain is rotated open and sterically trapped by the presence of nsp13.2. The nsp13.1 is therefore unable to engage with the downstream 5 't-RNA and is inactive. The RdRp is able to elongate freely in the downstream

557 direction.

558 Nsp13.1-swiveled (17\%): The rotation of the nsp13.1 protomer away from nsp13.2 provides space for the nsp13.1 1B domain to open and/or close. We therefore propose that nsp13.1-swiveled represents a transition state between the 1B-open (elongating) and nsp13.1-engaged (backtracking) states.

562 Nsp13.1-engaged (33\%): The nsp13.1 1B and RecA domains are clamped onto the 563 downstream 5'-t-RNA. In this state, nsp13.1 can translocate on the t-RNA in the 5'-3' 564 direction (shown by the orange arrow). This counteracts RdRp elongation and causes 565 backtracking (backward motion of the RdRp on the RNA, shown by the gray arrow 566 pointing upstream).

567 Also see Fig. S7 and Videos S1 and S2. 
569

570

571

572

573

574

575

576

577

578

579

580

581

582

583

584

585

586

587

588

589

590

591

592

593

594

595

596

597

598

599

600

601

602

603

604

605

606

607

608

609

610

611

\section{METHODS}

No statistical methods were used to predetermine sample size. The experiments were not randomized, and the investigators were not blinded to allocation during experiments and outcome assessment.

\section{Protein expression and purification}

SARS-CoV-2 nsp12 was expressed and purified as described ${ }^{20}$. Briefly, a pRSFDuet-1 plasmid containing $\mathrm{His}_{6}-\mathrm{SUMO}$ SARS-CoV-2 nsp12 (Addgene \#159107) was transformed into $E$. coli BL21-CodonPlus cells (Agilent). Cells were grown and protein expression was induced by the addition of isopropyl $\beta$-d-1-thiogalactopyranoside (IPTG). Cells were collected and lysed in a French press (Avestin). The lysate was cleared by centrifugation and purified on a HiTrap Heparin HP column (Cytiva). The fractions containing nsp12 were loaded onto a HisTrap HP column (Cytiva) for further purification. Eluted nsp12 was dialyzed, cleaved with His 6 -Ulp1 SUMO protease, and passed through a HisTrap HP column to remove the SUMO protease. Flow-through was collected, concentrated by centrifugal filtration (Amicon), and loaded on a Superdex 200 Hiload 16/600 (Cytiva). Glycerol was added to the purified nsp12, aliquoted, flash-frozen with liquid $\mathrm{N}_{2}$, and stored at $-80^{\circ} \mathrm{C}$.

SARS-CoV-2 nsp7/8 was expressed and purified as described ${ }^{20}$. Briefly, the pCDFDuet-1 plasmid containing His 6 SARS-CoV-2 nsp7/8 (Addgene \#159092) was transformed into $E$. coli BL21 (DE3). Cells were grown and protein expression was induced by the addition of IPTG. Cells were collected and lysed in a French press (Avestin). The lysate was cleared by centrifugation and purified on a HisTrap HP column (Cytiva). Eluted nsp7/8 was dialyzed, cleaved with His 6 -Prescission Protease to cleave His 6 tag, and then passed through a HisTrap HP column to remove the protease (Cytiva). Flow-through was collected, concentrated by centrifugal filtration (Amicon), and loaded onto a Superdex 75 Hiload 16/600 (Cytiva). Glycerol was added to the purified $\mathrm{nsp} 7 / 8$, aliquoted, flash-frozen with liquid $\mathrm{N}_{2}$, and stored at $-80^{\circ} \mathrm{C}$.

SARS-CoV-2 nsp13 was expressed and purified as described ${ }^{20}$. Briefly, the pet28 plasmid containing His 6 SARS-CoV-2 nsp13 (Addgene \#159390) was transformed into E. coli Rosetta (DE3) (Novagen). Cells were grown and protein expression was induced by the addition of IPTG. Cells were collected and lysed in a French press (Avestin). The lysate was cleared by centrifugation and purified on a HisTrap HP column (Cytiva). Eluted nsp13 was dialyzed, cleaved with His $\mathrm{H}_{6}$-Prescission Protease, and then passed through a HisTrap HP column to remove protease (Cytiva). Flow-through was collected, concentrated by centrifugal filtration (Amicon), and loaded onto a Superdex 200 Hiload $16 / 600$ (Cytiva). Glycerol was added to the purified nsp13, aliquoted, flash-frozen with liquid $\mathrm{N}_{2}$, and stored at $-80^{\circ} \mathrm{C}$.

\section{Preparation of SARS-CoV-2 nsp13-replication/transcription complex (RTC) for} Cryo-EM. Cryo-EM samples of SARS-CoV-2 nsp13-RTC were prepared as described 20. Briefly, purified nsp12 and nsp7/8 were concentrated, mixed in a 1:3 molar ratio, and incubated for 20 min at $22^{\circ} \mathrm{C}$. Annealed RNA scaffold (Horizon Discovery, Ltd.) was 
612 added to the nsp $7 / 8 / 12$ mixture and incubated for $15 \mathrm{~min}$ at $22^{\circ} \mathrm{C}$. Sample was buffer 613 exchanged into cryo-EM buffer [20 mM HEPES pH 8.0, $150 \mathrm{mM}$ K-Acetate, $10 \mathrm{mM}$ $614 \mathrm{MgCl}_{2}, 2 \mathrm{mM}$ DTT] and further incubated for $20 \mathrm{~min}$ at $30^{\circ} \mathrm{C}$. The sample was purified 615 over a Superose 6 Increase 10/300 GL column (Cyriva) in cryo-EM buffer. The peak corresponding to nsp7/8/12/RNA complex was pooled and concentrated by centrifugal filtration (Amicon). Purified nsp13 was concentrated by centrifugal filtration (Amicon) and buffer exchanged into cryo-EM buffer. Buffer exchanged nsp13 was mixed with ADP (1 mM final) and $\mathrm{AlF}_{3}(1 \mathrm{mM}$ final) and then added to nsp7/8/12/RNA at a molar

Cryo-EM grid preparation. Prior to grid freezing, 3-([3cholamidopropyl]dimethylammonio)-2-hydroxy-1-propanesulfonate (CHAPSO, Anatrace) was added to the sample ( $8 \mathrm{mM}$ final), resulting in a final complex concentration of $8 \mu \mathrm{M}$. The final buffer condition for the cryo-EM sample was $20 \mathrm{mM}$ HEPES pH 8.0, $150 \mathrm{mM}$ K-Acetate, $10 \mathrm{mM} \mathrm{MgCl}_{2}, 2 \mathrm{mM}$ DTT, $1 \mathrm{mM}$ ADP, $1 \mathrm{mM} \mathrm{AIF}_{3}, 8 \mathrm{mM}$ CHAPSO. C-flat holey carbon grids (CF-1.2/1.3-4Au, EMS) were glow-discharged for $20 \mathrm{~s}$ prior to the application of $3.5 \mu \mathrm{L}$ of sample. Using a Vitrobot Mark IV (Thermo Fisher Scientific), grids were blotted and plunge-froze into liquid ethane with $90 \%$ chamber humidity at $4^{\circ} \mathrm{C}$.

Cryo-EM data acquisition and processing. Structural biology software was accessed through the SBGrid consortium ${ }^{44}$. Grids were imaged using a $300 \mathrm{kV}$ Titan Krios (Thermo Fisher Scientific) equipped with a GIF BioQuantum and K3 camera (Gatan). Images were recorded with Leginon ${ }^{45}$ with a pixel size of $1.07 \AA / p x$ (micrograph dimension of $5760 \times 4092 \mathrm{px}$ ) over a defocus range of $-0.8 \mu \mathrm{m}$ to $-2.5 \mu \mathrm{m}$ with a $20 \mathrm{eV}$ slit. Movies were recorded in "counting mode" (native K3 camera binning 2) with 30 e$/ \mathrm{px} / \mathrm{s}$ in dose-fractionation mode with subframes of $50 \mathrm{~ms}$ over a $2.5 \mathrm{~s}$ exposure (50 frames) to give a total dose of $\sim 66$ e-/ $\AA^{2}$. Dose-fractionated movies were gainnormalized, drift-corrected, summed, and dose-weighted using MotionCor2 ${ }^{46}$. The contrast transfer function (CTF) was estimated for each summed image using the Patch CTF module in cryoSPARC v2.15.0 ${ }^{47}$. Particles were picked and extracted from the dose-weighted images with box size of 256 px using cryoSPARC Blob Picker and Particle Extraction. The entire dataset consisted of 17,806 motion-corrected images with $3,750,107$ particles. Particles were sorted using two rounds of cryoSPARC 2D classification ( $N=100$, where $N$ equals the number of classes), resulting in 661,105 curated particles that were re-extracted with a boxsize of 320 px. An initial model was generated using cryoSPARC $A b$ initio Reconstruction $(\mathrm{N}=3)$ on a subset of the particles. Particles were further curated using this initial model as a 3D template for cryoSPARC Heterogeneous Refinement ( $\mathrm{N}=3)$, resulting in 451,760 particles (green map, Fig. S1). Curated particles were further classified using cryoSPARC Heterogeneous Refinement $(\mathrm{N}=3)$. Each of the resulting $3 \mathrm{D}$ classes were further processed with cryoSPARC $A b$ initio Reconstruction ( $N=3$ ), generating three distinct models that could be used to sort particles [Ref 1: nsp131-RTC, Ref 2: nsp132-RTC, Ref 3: (nsp132-RTC) 2 . Using Ref $1-3$ as 3D templates for Heterogeneous Refinement $(N=6)$, multi-reference classification was performed on the 451,760 curated particles. Classification revealed 
657 three unique classes: nsp13 1 -RTC (class1; 85,206 particles; yellow), nsp13 2 -RTC

658 (class2-4; 315,216 particles; red), and (nsp132-RTC)2 (class5; 35,403 particles; blue).

659 Particles within each class were further processed using RELION 3.1-beta Bayesian

660 Polishing ${ }^{48}$. Polished particles were refined using cryoSAPRC Local and Global CTF

661 Refinement in combination with cryoSPARC Non-uniform Refinement ${ }^{49}$, resulting in

662 structures with the following particle counts and nominal resolutions: nsp13 1 -RTC

663 (85,187 particles; $3.2 \AA)$, nsp132-RTC (315,120 particles; $2.9 \AA)$, (nsp132-RTC) $2(35,392$

664

665

666

667

668

669

670

671

672

673

674

675

676

677

678

679

680

681

682

683

684

685

686

687

688

689

690

691

692

693

694

695

696

697

698

699

700

701 particles; $3.3 \AA$ ). To facilitate model building of nsp132-RTC, particles from nsp13 1 -RTC and nsp132-RTC were combined in a cryoSPARC Non-uniform Refinement, subtracted (masking the RTC), and further refined with cryoSPARC Local Refinement using a mask encompassing the RTC. The resulting map, deemed RTC (local), had nominal resolution of $2.8 \AA$. Additionally, particles from the nsp132-RTC were subtracted in different regions (using separate masks for nsp12-NiRAN, nsp13.1, and nsp13.2) and the particles from each subtraction were further refined with masked cryoSPARC Local Refinement. The resulting maps had the following nominal resolutions: nsp13.1(local): $3.4 \AA$, nsp13.2(local): $3.3 \AA$, nsp12-NiRAN(local): $2.7 \AA$. Locally refined maps were combined into an nsp132-RTC composite map using PHENIX 'Combine Focused Maps' 50,51 , with resulting nominal resolution of $2.8 \AA$. The nsp13-RecA domains in particles from the nsp131-RTC and nsp132-RTC classes were sorted using particle subtraction (masking around the RecA domains, shown as red mesh in Fig. S1), followed by masked RELION 3D classification. Classification of RecA domains in the nsp131-RTC particles (pale yellow) did not reveal discrete conformational heterogeneity in the RecA domains. However, classification of RecA domains in the nsp132-RTC particles (light red) revealed unique conformations of the RecA domains with the following particle counts and nominal resolutions: RecA classl (52,403 particles; $3.5 \AA)$, RecA class II (102,615 particles; $3.1 \AA$ A), RecA class III (54,830 particles; $3.5 \AA$ ), RecA class IV (105,272 particles; $3.1 \AA$ ). Local resolution calculations were generated using blocres and blocfilt from the Bsoft package ${ }^{29}$.

Model building and refinement. For an initial model of the nsp13 2 -RTC, the initial RTC model was derived from PDB 6XEZ ${ }^{20}$ and the initial nsp13 model from PDB 6ZSL ${ }^{32}$. The models were manually fit into the cryo-EM density maps using Chimera ${ }^{52}$ and rigidbody and real-space refined using Phenix real-space-refine ${ }^{50,51}$. For real-space refinement, rigid body refinement was followed by all-atom and B-factor refinement with Ramachandran and secondary structure restraints. Models were inspected and modified in Coot ${ }^{53}$.

\section{Molecular dynamics simulations}

General simulation setup and parameterization. Proteins, ATP, ADP, and ions were parameterized with the DES-Amber SF1.0 force field ${ }^{54}$. RNAs were parameterized with the Amber ff14 RNA force field ${ }^{55}$ with modified electrostatic, van der Waals, and torsional parameters to more accurately reproduce the energetics of nucleobase stacking ${ }^{56}$. The systems were solvated with water parameterized with the TIP4P-D water model ${ }^{57}$ and neutralized with a $150 \mathrm{mM} \mathrm{NaCl}$ buffer. The systems each contained $\sim 160,000$ atoms in a $110 \times 110 \times 110 \AA$ cubic box. 
Systems were first equilibrated on GPU Desmond using a mixed NVT/NPT schedule ${ }^{58}$, followed by a $1 \mu$ s relaxation simulation on Anton, a special-purpose machine for molecular dynamics simulations ${ }^{59}$. All production simulations were performed on Anton and initiated from the last frame of the relaxation simulation. Production simulations were performed in the NPT ensemble ${ }^{60}$ at $310 \mathrm{~K}$ using the Martyna-Tobias-Klein barostat ${ }^{61}$. The simulation time step was $2.5 \mathrm{fs}$, and a modified rRESPA integrator ${ }^{62}$ was used in which long-range electrostatic interactions were evaluated every three time steps. Electrostatic forces were calculated using the $u$-series method ${ }^{63}$. A 9-A cutoff was applied for the van der Waals calculations.

System preparation. The initial conformations of Class II nsp13.1 bound to the various substrates (ATPMg ${ }^{2+} / \mathrm{RNA}$ ADPMg ${ }^{2+} / \mathrm{RNA}$ ATPMg ${ }^{2+}$, and $\mathrm{ADPMg}{ }^{2+}$ ) were prepared based on the cryo-EM structure of the Class II nsp132-BTC ${ }_{5}$. The initial conformation of the Class IV, 1B-open nsp13.1 structure was prepared from the cryo-EM Class IV nsp13 ${ }_{2}-\mathrm{BTC}_{5}$ structure. $\mathrm{AlF}_{3}$ was removed from the active site. Missing loops and termini in proteins were capped with ACE/NME capping groups. In simulations with ATP at the active site, ATP was manually placed using ADP in the cryo-EM structure as the reference. The systems were prepared for simulation using the Protein Preparation Wizard in Schrödinger Maestro (Schrödinger Release 2020-4: Maestro, Schrödinger, LLC, New York, NY, 2020).

Simulation analysis. The average rmsd was calculated for the RecA2 domain (residues 450-690) and 1B domain (residues 145-200) of nsp13.1 between the cryo-EM structures and instantaneous structures from the trajectories, aligned on the RecA1 lobe (residues 240-440). Simulation structures shown in figures were rendered using PyMol (The PyMOL Molecular Graphics System, Version 2.0 Schrödinger, LLC).

Quantification and statistical analysis. The local resolution of the cryo-EM maps (Figs S3 and S5) was estimated using blocres ${ }^{29}$ with the following parameters: box size 15, sampling 1.1, and cutoff 0.5. Directional 3DFSCs (Figs. S3 and S5) were calculated using 3DFSC ${ }^{64}$. The quantification and statistical analyses for model refinement and validation were generated using MoIProbity ${ }^{65}$ and PHENIX ${ }^{51}$.

Data and code availability. All unique/stable reagents generated in this study are available without restriction from one of the Lead Contacts, Seth A. Darst (darst@rockefeller.edu). The cryo-EM density maps and atomic coordinates have been deposited in the EMDataBank and Protein Data Bank as follows: nsp131-RTC (EMD24431, 7RE2), nsp132-RTC (composite) (EMD-24430, 7RE1), (nsp132-RTC) 2 (EMD24432, 7RE3), nsp132-RTC (nsp13.1-apo) (EMD-24428, 7RDZ), nsp132-RTC (nsp13.1engaged) (EMD-24427, 7RDY), nsp132-RTC (nsp13.1-swiveled) (EMD-24429, 7RE0), nsp132-RTC (1B-open) (EMD-24426, 7RDX). The MD trajectories described in this work are available at 


\section{REFERENCES}

1. Wu, F. et al. A new coronavirus associated with human respiratory disease in China. Nature 579, 265-269 (2020).

749 2. Zhou, P. et al. A pneumonia outbreak associated with a new coronavirus of probable 750 bat origin. Nature 579, 270-273 (2020).

3. Subissi, L. et al. One severe acute respiratory syndrome coronavirus protein complex integrates processive RNA polymerase and exonuclease activities. Proceedings of the National Academy of Sciences of the United States of America 111, E3900-9 (2014). bound to nsp7 and nsp8 co-factors. Nature Communications 10, 2342-9 (2019). (2020) doi:10.1038/s41586-020-2368-8.

758

759

760

761

762

763

764

765

766

767

768

769

770

771

772

773

774

775

776

777

6. Gordon, C. J. et al. Remdesivir is a direct-acting antiviral that inhibits RNA-dependent RNA polymerase from severe acute respiratory syndrome coronavirus 2 with high potency. J Biol Chem 295, 6785-6797 (2020).

7. Tchesnokov, E. P. et al. Template-dependent inhibition of coronavirus RNAdependent RNA polymerase by remdesivir reveals a second mechanism of action. $J$ Biol Chem 295, 16156-16165 (2020).

8. Kokic, G. et al. Mechanism of SARS-CoV-2 polymerase stalling by remdesivir. Nat Commun 12, 279 (2021).

9. Snijder, E. J., Decroly, E. \& Ziebuhr, J. The Nonstructural Proteins Directing Coronavirus RNA Synthesis and Processing. Adv Virus Res 96, 59-126 (2016).

10. Dinten, L. C. van, Tol, H. van, Gorbalenya, A. E. \& Snijder, E. J. The Predicted Metal-Binding Region of the Arterivirus Helicase Protein Is Involved in Subgenomic mRNA Synthesis, Genome Replication, and Virion Biogenesis. J Virol 74, 5213-5223 (2000).

\section{Seybert, A., Dinten, L. C. van, Snijder, E. J. \& Ziebuhr, J. Biochemical}

Characterization of the Equine Arteritis Virus Helicase Suggests a Close Functional Relationship between Arterivirus and Coronavirus Helicases. J Virol 74, 9586-9593 (2000).

12. Seybert, A. et al. A Complex Zinc Finger Controls the Enzymatic Activities of Nidovirus Helicases. J Virol 79, 696-704 (2005). 
13. Lehmann, K. C., Snijder, E. J., Posthuma, C. C. \& Gorbalenya, A. E. What we know but do not understand about nidovirus helicases. Virus Res 202, 12-32 (2015).

14. Saikrishnan, K., Powell, B., Cook, N. J., Webb, M. R. \& Wigley, D. B. Mechanistic basis of 5'-3' translocation in SF1B helicases. Cell 137, 849-59 (2009).

15. Adedeji, A. O. et al. Mechanism of Nucleic Acid Unwinding by SARS-CoV Helicase. Plos One 7, e36521 (2012).

16. Mickolajczyk, K. J. et al. Force-dependent stimulation of RNA unwinding by SARSCoV-2 nsp13 helicase. Biophys J (2020) doi:10.1016/j.bpj.2020.11.2276.

17. Singleton, M. R., Dillingham, M. S. \& Wigley, D. B. Structure and Mechanism of Helicases and Nucleic Acid Translocases. Annu Rev Biochem 76, 23-50 (2007).

18. Hao, W. et al. Crystal structure of Middle East respiratory syndrome coronavirus helicase. Plos Pathog 13, e1006474 (2017).

19. Jia, Z. et al. Delicate structural coordination of the Severe Acute Respiratory Syndrome coronavirus Nsp13 upon ATP hydrolysis. Nucleic Acids Res 47, 6538-6550 (2019).

20. Chen, J. et al. Structural basis for helicase-polymerase coupling in the SARS-CoV-2 replication-transcription complex. Cell 182, 1560-1573.e13 (2020).

21. Yan, L. et al. Architecture of a SARS-CoV-2 mini replication and transcription complex. Nat Commun 11, 5874 (2020).

22. Yan, L. et al. Cryo-EM Structure of an Extended SARS-CoV-2 Replication and Transcription Complex Reveals an Intermediate State in Cap Synthesis. Cell 184, 184193.e10 (2021).

23. Deng, Z. et al. Structural basis for the regulatory function of a complex zinc-binding domain in a replicative arterivirus helicase resembling a nonsense-mediated mRNA decay helicase. Nucleic Acids Res 42, 3464-77 (2013).

24. Tavares, R. de C. A., Mahadeshwar, G., Wan, H., Huston, N. C. \& Pyle, A. M. The Global and Local Distribution of RNA Structure throughout the SARS-CoV-2 Genome. J Virol 95, (2021).

25. Huston, N. C. et al. Comprehensive in vivo secondary structure of the SARS-CoV-2 genome reveals novel regulatory motifs and mechanisms. Mol Cell 81, 584-598.e5 (2021).

26. Malone, B. et al. Structural basis for backtracking by the SARS-CoV-2 replicationtranscription complex. Proc National Acad Sci 118, e2102516118 (2021). 
811 27. Seifert, M. et al. Signatures and mechanisms of efficacious therapeutic

812 ribonucleotides against SARS-CoV-2 revealed by analysis of its replicase using

813 magnetic tweezers. Biorxiv 2020.08.06.240325 (2020) doi:10.1101/2020.08.06.240325.

814 28. Scheres, S. H. W. RELION: implementation of a Bayesian approach to cryo-EM structure determination. Journal of structural biology 180, 519-530 (2012).

29. Cardone, G., Heymann, J. B. \& Steven, A. C. One number does not fit all: mapping local variations in resolution in cryo-EM reconstructions. Journal of structural biology 184, 226-236 (2013).

30. Bai, X., Rajendra, E., Yang, G., Shi, Y. \& Scheres, S. H. W. Sampling the (2015).

31. Yan, L. et al. Coupling of N7-methyltransferase and 3'-5' exoribonuclease with SARS-CoV-2 polymerase reveals mechanisms for capping and proofreading. Cell 184, 3474-3485.e11 (2021).

32. Newman, J. A. et al. Structure, Mechanism and Crystallographic fragment screening of the SARS-CoV-2 NSP13 helicase. Biorxiv 2021.03.15.435326 (2021) doi:10.1101/2021.03.15.435326.

33. Chakrabarti, S. et al. Molecular mechanisms for the RNA-dependent ATPase activity of Upf1 and its regulation by Upf2. Mol Cell 41, 693-703 (2011).

34. Cheng, Z., Muhlrad, D., Lim, M. K., Parker, R. \& Song, H. Structural and functional insights into the human Upf1 helicase core. Embo J 26, 253-264 (2007).

35. Dillingham, M. S., Wigley, D. B. \& Webb, M. R. Demonstration of Unidirectional Single-Stranded DNA Translocation by PcrA Helicase: Measurement of Step Size and Translocation Speed †. Biochemistry-us 39, 205-212 (2000).

36. Lohman, T. M., Tomko, E. J. \& Wu, C. G. Non-hexameric DNA helicases and translocases: mechanisms and regulation. Nat Rev Mol Cell Bio 9, 391-401 (2008). Stepping Mechanism for E. coli UvrD Monomer Translocation along Single-Stranded DNA. Mol Cell 26, 335-347 (2007). inchworm mechanism. Cell 97, 75-84 (1999). 
40. Minskaia, E. et al. Discovery of an RNA virus 3' $->5$ ' exoribonuclease that is critically involved in coronavirus RNA synthesis. Proc National Acad Sci 103, 5108-5113 (2006).

41. Gorbalenya, A. E., Enjuanes, L., Ziebuhr, J. \& Snijder, E. J. Nidovirales: Evolving the largest RNA virus genome. Virus Res 117, 17-37 (2006).

42. Denison, M. R., Graham, R. L., Donaldson, E. F., Eckerle, L. D. \& Baric, R. S. Coronaviruses: An RNA proofreading machine regulates replication fidelity and diversity. Rna Biol 8, 270-279 (2011).

43. Agostini, M. L. et al. Coronavirus Susceptibility to the Antiviral Remdesivir (GS5734) Is Mediated by the Viral Polymerase and the Proofreading Exoribonuclease. Mbio 9, e00221-18 (2018).

44. Morin, A. et al. Collaboration gets the most out of software. eLife 2, e01456 (2013).

45. Suloway, C. et al. Automated molecular microscopy: the new Leginon system. Journal of structural biology 151, 41-60 (2005).

46. Zheng, S. Q. et al. MotionCor2: anisotropic correction of beam-induced motion for improved cryo-electron microscopy. Nature methods 14, 331-332 (2017).

47. Punjani, A., Rubinstein, J. L., Fleet, D. J. \& Brubaker, M. A. cryoSPARC: algorithms for rapid unsupervised cryo-EM structure determination. Nat Methods 14, 290-296 (2017).

48. Zivanov, J. et al. New tools for automated high-resolution cryo-EM structure determination in RELION-3. eLife 7, (2018).

49. Punjani, A., Zhang, H. \& Fleet, D. J. Non-uniform refinement: adaptive regularization improves single-particle cryo-EM reconstruction. Nat Methods 17, 1214-1221 (2020).

50. Afonine, P. V. et al. New tools for the analysis and validation of cryo-EM maps and atomic models. Acta crystallographica. Section D, Structural biology 74, 814-840 (2018).

51. Adams, P. D. et al. PHENIX: a comprehensive Python-based system for macromolecular structure solution. Acta Crystallographica Section D Biological Crystallography 66, 213-221 (2010).

52. Pettersen, E. F. et al. UCSF Chimera--a visualization system for exploratory research and analysis. Journal of computational chemistry 25, 1605-1612 (2004).

53. Emsley, P. \& Cowtan, K. Coot: model-building tools for molecular graphics. Acta Crystallographica Section D Biological Crystallography 60, 2126-2132 (2004). 
54. Piana, S., Robustelli, P., Tan, D., Chen, S. \& Shaw, D. E. Development of a Force Field for the Simulation of Single-Chain Proteins and Protein-Protein Complexes. $J$ Chem Theory Comput 16, 2494-2507 (2020).

55. Maier, J. A. et al. ff14SB: Improving the Accuracy of Protein Side Chain and Backbone Parameters from ff99SB. J Chem Theory Comput 11, 3696-3713 (2015).

56. Tan, D., Piana, S., Dirks, R. M. \& Shaw, D. E. RNA force field with accuracy comparable to state-of-the-art protein force fields. Proc National Acad Sci 115, 201713027 (2018).

57. Piana, S., Donchev, A. G., Robustelli, P. \& Shaw, D. E. Water Dispersion Interactions Strongly Influence Simulated Structural Properties of Disordered Protein States. J Phys Chem B 119, 5113-5123 (2015).

58. Bowers, K. J. et al. Scalable Algorithms for Molecular Dynamics Simulations on Commodity Clusters. Acm leee Sc 2006 Conf Sc'06 43-43 (2006) doi:10.1109/sc.2006.54.

59. Shaw, D. E. et al. Anton 2: Raising the Bar for Performance and Programmability in a Special-Purpose Molecular Dynamics Supercomputer. Sc14 Int Conf High Perform Comput Netw Storage Analysis 41-53 (2014) doi:10.1109/sc.2014.9.

60. Lippert, R. A. et al. Accurate and efficient integration for molecular dynamics simulations at constant temperature and pressure. J Chem Phys 139, 164106 (2013).

61. Martyna, G. J., Tobias, D. J. \& Klein, M. L. Constant pressure molecular dynamics algorithms. J Chem Phys 101, 4177-4189 (1994).

62. Tuckerman, M., Berne, B. J. \& Martyna, G. J. Reversible multiple time scale molecular dynamics. J Chem Phys 97, 1990-2001 (1992).

63. Predescu, C. et al. The u -series: A separable decomposition for electrostatics computation with improved accuracy. J Chem Phys 152, 084113 (2020).

64. Tan, Y. Z. et al. Addressing preferred specimen orientation in single-particle cryoEM through tilting. Nature methods 14, 793-796 (2017).

65. Chen, V. B. et al. MolProbity: all-atom structure validation for macromolecular crystallography. Acta Crystallographica Section D Biological Crystallography 66, 12-21 (2010). 